\title{
Magnetic collapse and the behavior of transition metal oxides at high pressure
}

\author{
I. Leonov, ${ }^{1,2}$ L. Pourovskii, ${ }^{2,3,4}$ A. Georges, ${ }^{3,4,5}$ and I. A. Abrikosov ${ }^{2,6}$ \\ ${ }^{1}$ Theoretical Physics III, Center for Electronic Correlations and Magnetism, Institute of Physics, \\ University of Augsburg, D-86135 Augsburg, Germany \\ ${ }^{2}$ Materials Modeling and Development Laboratory, National University of Science and Technology “MISIS”, Moscow 119049, Russia \\ ${ }^{3}$ Centre de Physique Théorique, École Polytechnique, CNRS, Université Paris-Saclay, F-91128 Palaiseau, France \\ ${ }^{4}$ Collège de France, 11 place Marcelin Berthelot, F-75005 Paris, France \\ ${ }^{5}$ DQMP, Université de Genève, 24 quai Ernest Ansermet, CH-1211 Genève, Switzerland \\ ${ }^{6}$ Department of Physics, Chemistry and Biology (IFM), Linköping University, SE-58183 Linköping, Sweden \\ (Received 1 August 2016; revised manuscript received 12 September 2016; published 20 October 2016)
}

\begin{abstract}
We report a detail theoretical study of the electronic structure and phase stability of transition metal oxides $\mathrm{MnO}, \mathrm{FeO}, \mathrm{CoO}$, and $\mathrm{NiO}$ in their paramagnetic cubic $B 1$ structure by employing dynamical mean-field theory of correlated electrons combined with ab initio band-structure methods. Our calculations reveal that under pressure these materials exhibit a Mott insulator-metal transition (IMT) which is accompanied by a simultaneous collapse of local magnetic moments and lattice volume, implying a complex interplay between chemical bonding and electronic correlations. Moreover, our results for the transition pressure show a monotonous decrease from $\sim 145$ to $40 \mathrm{GPa}$, upon moving from $\mathrm{MnO}$ to $\mathrm{CoO}$. In contrast to that, in $\mathrm{NiO}$, magnetic collapse is found to occur at a remarkably higher pressure of $\sim 429 \mathrm{GPa}$. We provide a unified picture of such a behavior and suggest that it is primarily a localized to itinerant moment behavior transition at the IMT that gives rise to magnetic collapse in transition metal oxides.
\end{abstract}

DOI: 10.1103/PhysRevB.94.155135

\section{INTRODUCTION}

The effect of strong electron correlations plays a crucial role in the formation of a variety of electronic and magnetic phenomena experimentally observed in transition metal oxides [1]. The pressure-induced Mott insulator-metal transition (IMT) [2] is of particular importance for both fundamental science and modern technology, for example, due to its potential application in micro- and optoelectronics. Moreover, transition metals $\mathrm{Mn}, \mathrm{Co}$, and $\mathrm{Ni}$ are known to be siderophile elements, i.e., together with iron, they are extensively abundant in the Earth's interior [3]. Because of that, the electronic structure and phase stability of these basic oxide compounds, especially in the high-pressure and temperature range, is of fundamental importance for understanding the properties and evolution of the Earth's lower mantle [4].

It has been shown that the pressure-induced Mott insulatormetal transition in these compounds is followed by a magnetic collapse, a remarkable reduction of magnetic moments of transition metal ions [5,6], which strongly influences their electronic and structural properties. Since our present knowledge of the Earth's interior structure is mainly based on average geophysical observations, e.g., from seismology, quantifying the effects of magnetic collapse on the electronic and structural properties of these minerals is of particular interest for geophysics. While the properties can be calculated employing, e.g., standard band-structure methods, these techniques cannot capture all the generic aspects of a Mott IMT, such as a formation of the lower- and upper-Hubbard bands, coherent quasiparticle behavior, strong renormalization of the electron mass, etc., because of the neglect of the effect of strong correlations of localized $3 d$ electrons. Therefore, these methods have only limited applicability and cannot provide a quantitative description of the electronic and lattice properties. In particular, at low pressures they predict a metallic behavior for $\mathrm{FeO}$ and $\mathrm{CoO}$, which are, in fact, insulators $[5,7,8]$. This obstacle can be overcome by employing, e.g., a state-of-the-art method for calculating the electronic structure of strongly correlated materials [density functional plus dynamical mean-field theory (DFT+DMFT)] $[9,10]$. It merges ab initio band-structure techniques, such as the local density approximation (LDA) or the generalized gradient approximation (GGA), with dynamical mean-field theory (DMFT) of correlated electrons [9], providing a good quantitative description of the electronic and lattice properties [11-28]. In particular, this advanced theory makes it possible to determine the electronic structure and phase stability of paramagnetic correlated materials at finite temperatures, e.g., near a Mott insulator-metal transition [13-15,20-28]. The DFT+DMFT approach has been used to study the electronic and structural properties of correlated electron materials [1120], including transition metal monoxides [22-29]. In practice, however, these calculations employed different approximations, resulting in various scenarios for the IMT. For example, a local moment collapse at the IMT was found to occur in $\mathrm{MnO}$ [22]; for $\mathrm{FeO}$, different calculations predicted either a Mott transition in the high-spin state followed by a slow crossover into the low-spin one [24] or the complete absence of magnetic collapse [23]. In addition, most of these theoretical calculations used experimental equations of state, neglecting the effects of coupling between the electrons and lattice at the IMT $[23,24,26]$.

In this paper, we employ a state-of-the-art fully selfconsistent in charge density DFT+DMFT approach [30] to explore the electronic structure, magnetic state, and phase stability of all late transition metal monoxides, from $\mathrm{MnO}$ to $\mathrm{NiO}$. We use this advanced theory to systematically study these oxides at high pressure and temperature in their paramagnetic cubic $B 1$ lattice structure. We find that in all four compounds the Mott insulator-metal transition is accompanied by a 
simultaneous collapse of local moments. Our results suggest that the magnetic collapse is driven by a transition from the localized to itinerant moment behavior at the IMT. The phase transition is of first order with a significant fractional volume collapse $\Delta V / V$ ranging from $8.6 \%$ to $13.6 \%$ for $\mathrm{MnO}, \mathrm{FeO}$, and $\mathrm{CoO}$, and it is only about $1.4 \%$ for $\mathrm{NiO}$. We predict a monotonous decrease of the transition pressure from $\mathrm{MnO}$ to $\mathrm{CoO}$, namely, from $\sim 145$ to $40 \mathrm{GPa}$, implying a complex interplay between chemical bonding and electronic correlations in these materials. In contrast to that, the Mott transition and magnetic collapse is predicted to occur at $\sim 429 \mathrm{GPa}$ in $\mathrm{NiO}$, i.e., it has a substantially higher value than that in the other monoxides, which can be understood as a crossover in the effective degeneracy of low-energy excitations from five-orbital (as in $\mathrm{MnO}, \mathrm{FeO}$, and $\mathrm{CoO}$ ) to two-orbital behavior (as in $\mathrm{NiO}$ ). Overall, our calculations provide a unified picture for the pressure evolution of all late transition metal monoxides, that of a first-order Mott transition accompanied by a simultaneous collapse of local magnetic moments.

\section{METHOD}

Here, we investigate the electronic and structural properties of correlated transition metal oxides $\mathrm{MnO}, \mathrm{FeO}, \mathrm{CoO}$, and $\mathrm{NiO}$ at high pressure and temperature using the GGA+DMFT computational approach. To this end, we employ a fully self-consistent in charge density DFT+DMFT scheme [30] implemented with plane-wave pseudopotentials [31] to calculate the electronic structure, magnetic state, and phase stability of these compounds. For the partially filled $\mathrm{Mn}, \mathrm{Fe}, \mathrm{Co}$, and Ni $3 d$ and $\mathrm{O} 2 p$ orbitals we construct a basis set of atomic-centered symmetry-constrained Wannier functions $[32,33]$. To solve the realistic many-body problem, we employ the continuous-time hybridization-expansion quantum Monte Carlo algorithm [34]. The calculations are performed for a cubic rocksalt $(B 1)$ crystal structure in the paramagnetic state at a temperature $T=$ $1160 \mathrm{~K}$. We use the following values of the average Hubbard $U$ and Hund's exchange $J$ as estimated [8,22-27,35] previously: $U=8.0 \mathrm{eV}$ and $J=0.86 \mathrm{eV}$ for the Mn $3 d$ orbitals, 7.0 and $0.89 \mathrm{eV}$ for $\mathrm{Fe}, 8.0$ and $0.9 \mathrm{eV}$ for $\mathrm{Co}$, and 10.0 and $1.0 \mathrm{eV}$ for $\mathrm{Ni}$, respectively. The Coulomb interaction has been treated in the density-density approximation. The spin-orbit coupling was neglected in these calculations. Moreover, the $U$ and $J$ values are assumed to remain constant upon variation of the lattice volume. We employ the fully localized double counting correction, evaluated from the self-consistently determined local occupations, to account for the electronic interactions already described by GGA. The spectral functions were computed using the maximum entropy method.

\section{RESULTS AND DISCUSSION}

Our results for the local moment versus volume and pressure equations of state are summarized in Fig. 1. To this end, we calculate the total energy and fluctuating (instantaneous) local moment $\sqrt{\left\langle m_{z}^{2}\right\rangle}$ for these compounds as a function of volume. We evaluate pressure by fitting our results for the total energy to the third-order Birch-Murnaghan equation of states separately for the low- and high-volume regions. We
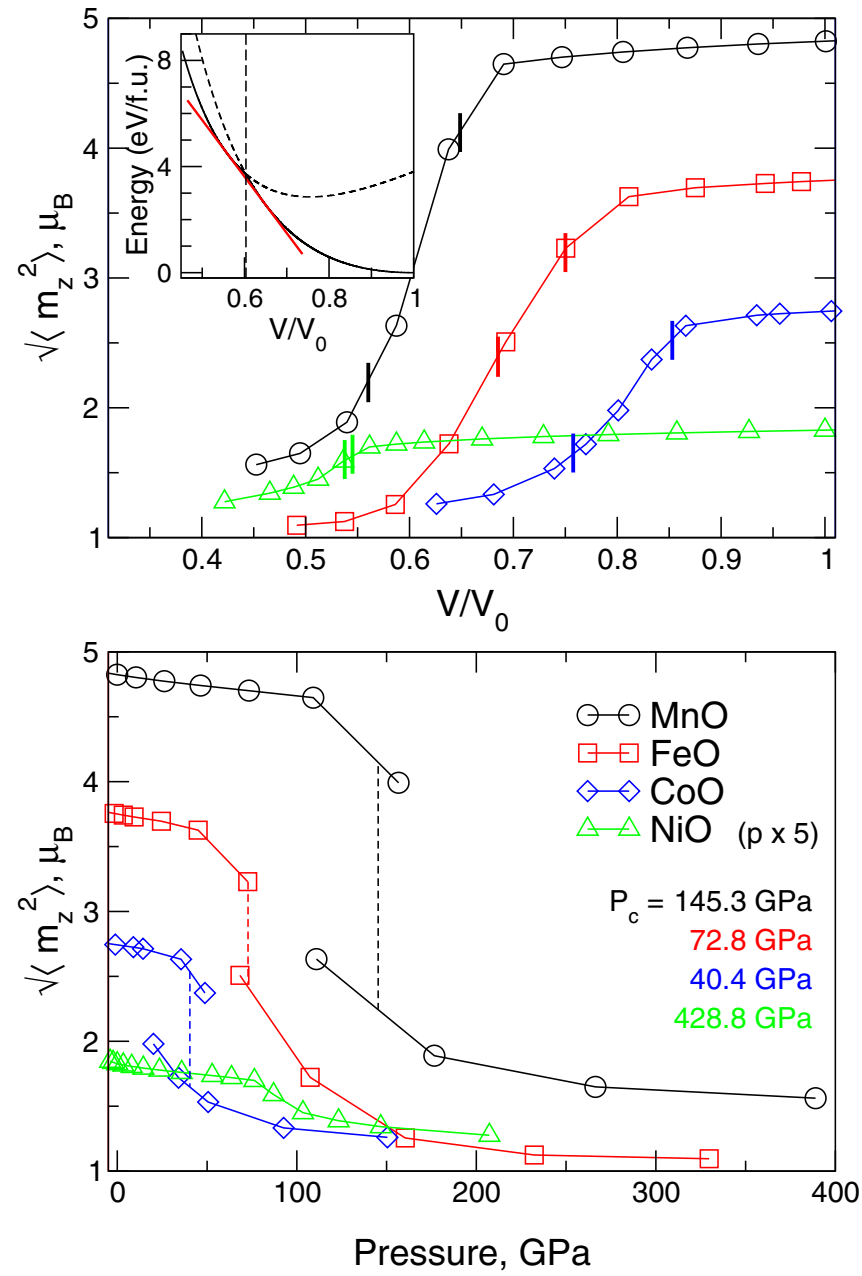

FIG. 1. Local moment $\sqrt{\left\langle m_{z}^{2}\right\rangle}$ calculated by DFT+DMFT as a function of lattice volume (top) and pressure (bottom). The IMT associated with the lattice volume collapse is shown by vertical solid (top) and dashed lines (bottom). The inset shows the total energy calculated by DFT+DMFT and the Maxwell construction for paramagnetic $\mathrm{MnO}$. Note that for $\mathrm{NiO}$ the pressure scale is shrunk by a factor of 5 .

obtain that all four compounds exhibit magnetic collapse-a remarkable reduction of the local magnetic moments upon compression of the lattice. Indeed, the local moments are seen to retain their high-spin values, e.g., for $\mathrm{NiO}$ and $\mathrm{MnO}$ of $\sim 1.8 \mu_{B}-4.8 \mu_{B}$, respectively, upon compression down to about $0.6-0.7 \mathrm{~V} / V_{0}$. In fact, these values correspond to the high-spin magnetic state of the $\mathrm{Mn}^{2+}$ and $\mathrm{Ni}^{2+}$ ions. Thus, in a cubic crystal field, the $\mathrm{Mn}^{2+}\left(3 d^{5}\right.$ electronic configuration) and $\mathrm{Ni}^{2+}\left(3 d^{8}\right)$ ions have a local moment of $5 \mu_{B}$ and $2 \mu_{B}$, respectively. Upon further compression, a high-spin (HS) to low-spin (LS) crossover takes place in all four materials, with a collapse of the local moments to a LS state. The resulting low-spin local moments are about $1.6 \mu_{B}, 1.1 \mu_{B}, 1.26 \mu_{B}$, and $1.28 \mu_{B}$ for $\mathrm{MnO}, \mathrm{FeO}, \mathrm{CoO}$, and $\mathrm{NiO}$, respectively. In addition, upon compression, a substantial redistribution of electrons between the $t_{2 g}$ and $e_{g}$ orbitals is found within the transition metal $3 d$ bands of $\mathrm{MnO}, \mathrm{FeO}$, and $\mathrm{CoO}$ [shown for $\mathrm{MnO}$ in Fig. 2(b)]. Thus, the $t_{2 g}$ orbital occupations are 

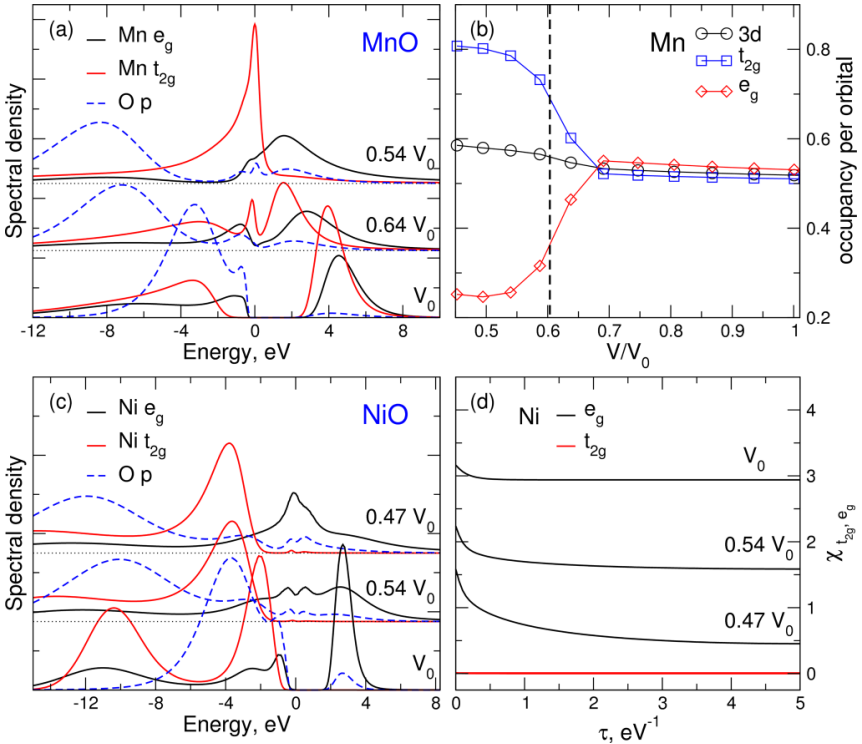

FIG. 2. Evolution of the spectral function (a) and orbital occupations (b) calculated by DFT+DMFT for paramagnetic $\mathrm{MnO}$ as a function of volume. Spectral function (c) and local spin-spin correlation function $\chi(\tau)=\left\langle\hat{m}_{z}(\tau) \hat{m}_{z}(0)\right\rangle(\mathrm{d})$ of paramagnetic NiO. $\tau$ is the imaginary time. At equilibrium volume $V_{0}$, the $3 d$ electrons are localized to form fluctuating moments $[\chi(\tau)$ is seen to be almost constant and close to its maximal value $S=1$ for the Ni $e_{g}$ states]. At high compression, the $3 d$ electrons show an itinerant magnetic behavior, implying a localized to itinerant moment crossover under pressure.

found to gradually increase with pressure, whereas the $e_{g}$ orbitals are strongly depopulated (below 0.27 for $\mathrm{MnO}$ and $\mathrm{FeO}$, and 0.44 for $\mathrm{CoO}$ ). The $3 d$ total occupancy remains essentially unchanged with pressure. We therefore interpret this spin crossover as a HS-LS transition.

Our results reveal that the magnetic transition pressures vary substantially among all these compounds. In particular, for $\mathrm{MnO}$, magnetic collapse is found to take place at a relatively high pressure of about $145 \mathrm{GPa}$ (see Table I) [36]. Moreover, for $\mathrm{FeO}$ and $\mathrm{CoO}$, it occurs at remarkably smaller values of about 73 and $40 \mathrm{GPa}$, respectively. That is, the transition pressure appears to monotonously decrease from $\mathrm{MnO}$ to $\mathrm{CoO}$, which implies a complex interplay between chemical bonding and electronic correlations in these materials. However, in contrast to that, $\mathrm{NiO}$ has a high transition pressure $\sim 429 \mathrm{GPa}$, which is considerably larger than that of $\mathrm{MnO}, \mathrm{FeO}$, and

TABLE I. Calculated structural parameters for the paramagnetic $B 1$ phase of transition metal oxides. $V_{0}$ is ambient pressure volume; $V_{\text {tr }}$ are the lattice volume collapse values; $K$, bulk modulus for the low/high-pressure phase; $K^{\prime} \equiv d K / d P$ is 4.1 for $\mathrm{MnO}, \mathrm{FeO}$, and $\mathrm{CoO} ; K^{\prime}=4.3$ for $\mathrm{NiO}$.

\begin{tabular}{lccccc}
\hline \hline Oxide & $P_{\mathrm{c}}(\mathrm{GPa})$ & $V_{0}\left(\right.$ a.u. $\left.^{3}\right)$ & $V_{\text {tr }}\left(\right.$ a.u. $\left.{ }^{3}\right)$ & $\Delta V / V(\%)$ & $K(\mathrm{GPa})$ \\
\hline $\mathrm{MnO}$ & 145.3 & 158.9 & $103.1 / 89.0$ & 13.6 & $137 / 263$ \\
$\mathrm{FeO}$ & 72.8 & 146.3 & $109.7 / 100.2$ & 8.6 & $140 / 162$ \\
$\mathrm{CoO}$ & 40.4 & 137 & $116.9 / 103.8$ & 11.2 & $184 / 246$ \\
$\mathrm{NiO}$ & 428.8 & 128 & $69.8 / 68.8$ & 1.4 & $187 / 188$ \\
\hline \hline
\end{tabular}
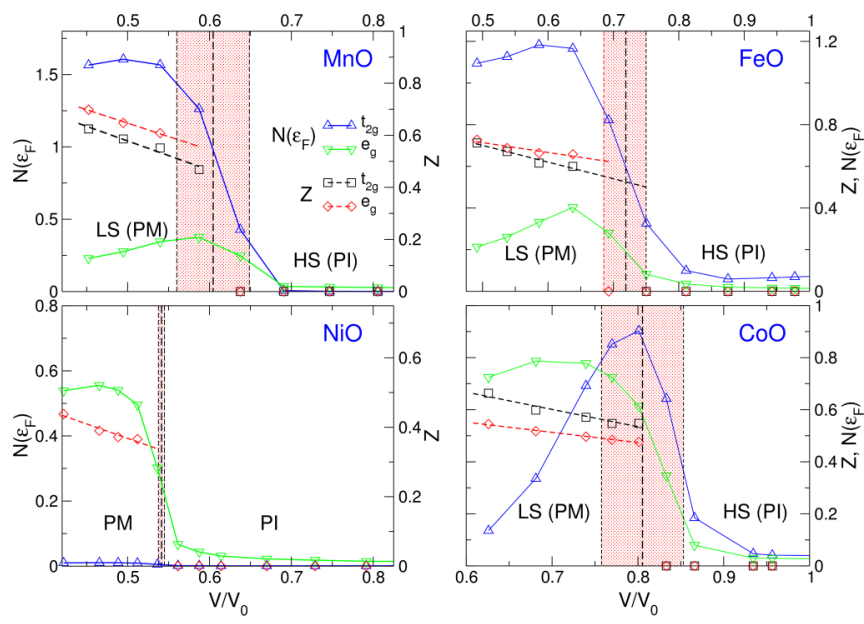

FIG. 3. Quasiparticle weight and spectral weight at the Fermi level calculated for the $t_{2 g}$ and $e_{g}$ orbitals as a function of lattice volume. Our result for the lattice volume collapse calculated by DFT+DMFT is marked by a red shaded rectangle. The quasiparticle weight $Z=[1-\partial \operatorname{Im} \Sigma(i \omega) / \partial i \omega]^{-1}$ is evaluated from the slope of the polynomial fit of the imaginary part of the self-energy $\Sigma\left(i \omega_{n}\right)$ at $\omega=0$.

CoO. This remarkable behavior can be understood as a continuous reduction of the strength of electronic correlations upon changing of the electron configuration from $3 d^{5}$ in $\mathrm{Mn}^{2+}$ ions to $3 d^{7}$ in $\mathrm{Co}^{2+}$. In fact, the effective interaction strength $U_{\text {eff }}=E\left(d^{n+1}\right)+E\left(d^{n-1}\right)-2 E\left(d^{n}\right)$, where $E$ is the ground-state energy for a $3 d$ shell of the corresponding occupancy, and, hence, the tendency towards localization of the $3 d$ electrons, changes from $U+4 J$ for $\mathrm{MnO}$ to $U-3 J$ for $\mathrm{CoO}$. On the other hand, it increases substantially in $\mathrm{NiO}$ due to a crossover in the effective degeneracy of low-energy excitations from five-orbital (as in $\mathrm{MnO}, \mathrm{FeO}$, and $\mathrm{CoO}$ ) to two-orbital behavior (as in $\mathrm{NiO}$ ).

Next, we address the spectral properties of paramagnetic transition metal oxides. In Figs. 2(a) and 2(c) we present our results for the evolution of the spectral function of $\mathrm{MnO}$ and $\mathrm{NiO}$ as a function of lattice volume. We note that at ambient pressure all these compounds (from $\mathrm{MnO}$ to $\mathrm{NiO}$ ) are Mott insulators with large $d$ - $d$ energy gaps, in agreement with experiment. This result is in remarkable contrast to that previously obtained by standard band-structure methods, which predict metallic behavior, e.g., for $\mathrm{FeO}$ and $\mathrm{CoO}$. This implies the crucial importance of strong correlations of localized $3 d$ electrons, to determine the electronic and magnetic properties of transition metal oxides. Indeed, at ambient pressure, the $3 d$ electrons are strongly localized, as it is seen from our result for the local spin susceptibility $\chi(\tau)=\left\langle\hat{m}_{z}(\tau) \hat{m}_{z}(0)\right\rangle$ [where $\tau$ is the imaginary time; see Fig. 2(d)]. Upon further compression, the $3 d$ electrons exhibit a crossover from localized to itinerant moment behavior which is associated with a Mott transition, as it is clearly seen in paramagnetic NiO. Upon the Mott transition, all these materials exhibit a strongly correlated metallic behavior with the formation of lower- and upper-Hubbard bands, and the quasiparticle peak at the Fermi level, associated with a substantial renormalization of the electron mass (see Fig. 3). 
We find that in all four compounds magnetic collapse is accompanied by a lattice collapse, which implies a complex interplay between electronic and lattice degrees of freedom at the transition. The phase transition is of first order with a significant from $8.6 \%$ to $13.6 \%$ fractional volume collapse, $\Delta V / V$, except for $\mathrm{NiO}$, where a resulting change of the lattice volume is about $1.4 \%$ (see Table I). We note, however, that these values should be considered as an upper-bound estimate because of neglecting by multiple intermediate-phase transitions upon fitting the total-energy result to the third-order Birch-Murnaghan equation of states. These findings may have important implications for geophysics. In particular, magnetic collapse should result in an anomalous behavior, e.g., of density and elasticity. On the other hand, variations of density and elastic moduli control the speed of seismic waves, playing a crucial role in the interpretation of seismological data [4]. Our results for the bulk modulus (see Table I) in the HS phase are remarkably smaller than that in the LS phase in all these compounds, implying an enhancement of the compressibility at the phase transition.

In addition, we evaluated the quasiparticle weights by using a polynomial fit of the imaginary part of the self-energy $\Sigma\left(i \omega_{n}\right)$ at the lowest Matsubara frequencies $\omega_{n}$ (shown in Fig. 3). These results suggest that magnetic collapse is accompanied by a simultaneous Mott metal-insulator transition and collapse of the lattice volume. The electronic effective mass diverges at the IMT, in accordance with a Brinkman-Rice picture [37] of the IMT. We note that this divergence coincides with the drop of the spectral weight for the $t_{2 g}$ and $e_{g}$ orbitals at the Fermi level, as shown in Fig. 3. Our findings clearly indicate the crucial importance of electronic correlations to explain the electronic structure and lattice properties of correlated transition metal oxides.

Overall, our calculations reveal that the pressure-induced Mott IMT is accompanied by a simultaneous local moment collapse and by a discontinuous change of the lattice volume. Moreover, under pressure, all these materials exhibit a continuous transformation from a localized to itinerant moment behavior of the $3 d$ electrons. This is in line with the classical case of the Mott transition, in which IMT concurs with a complete collapse of magnetism. We note that magnetic collapse is also found to occur in the paramagnetic $B 1$ phase of $\mathrm{NiO}$, which may have important implications for understanding of the actual driving force behind the magnetic collapse. In fact, the $\mathrm{Ni}^{2+}$ ion has a $3 d^{8}$ electron configuration with completely occupied $t_{2 g}$ and half-filled $e_{g}$ bands. Therefore, changes in the crystal field splitting with pressure cannot result in a magnetic collapse transition in $\mathrm{NiO}$. On the basis of these observations, we suggest that it is primarily a localized to itinerant moment behavior transition which occurs at the IMT that gives rise to magnetic collapse in transition metal oxides. The crystal field, however, governs the character of the magnetic collapse as well as the magnitude of the high- and low- spin moments at a given pressure. Moreover, our results for the magnetic collapse and discontinuous change of the lattice volume found in these compounds at high pressures may have important geophysical implications. In fact, these phenomena may affect the structure and seismic properties of the Earth's interior.

In conclusion, we determine the electronic structure and phase stability of paramagnetic $B 1$-structured transition metal oxides at high pressure and temperature. Our results reveal a pressure-induced Mott insulator-metal transition which is accompanied by a simultaneous collapse of local moments and lattice volume. Upon compression, we observe a continuous transformation from a localized to itinerant moment behavior of the $3 d$ electrons. We argue that the magnetic collapse is primarily due to a localized to itinerant moment behavior transition which occurs at the IMT. We point out the importance of further theoretical and experimental investigations of the behavior of transition metal oxides at the high-pressure and temperature range for a better understanding of the Earth's interior.

\section{ACKNOWLEDGMENTS}

We thank V. I. Anisimov and D. Vollhardt for valuable discussions. I.L. acknowledges support by the Deutsche Forschungsgemeinschaft through Transregio TRR 80 and the Ministry of Education and Science of the Russian Federation in the framework of Increase Competitiveness Program of NUST "MISIS" (K3-2016-027), implemented by a governmental decree dated 16th of March 2013, N 211. L.P. acknowledges computational resources provided by the Swedish National Infrastructure for Computing (SNIC) at the National Supercomputer Centre (NSC) and PDC Center for High Performance Computing. A.G. acknowledges the support of the European Research Council (ERC-319286 QMAC) and of the Swiss National Science Foundation (NCCR MARVEL). I.A.A. acknowledges the support from the Swedish Research Council (VR) Grant No. 2015-04391, the Swedish Foundation for Strategic Research (SSF) Grant No. SRL 10-0026, the Knut and Alice Wallenberg Foundation through Grant No. 2014-2019, and the Swedish Government Strategic Research Area Grant Swedish e-Science Research Centre (SeRC). The support from Ministry of Education and Science of the Russian Federation (Grant No. 14.Y26.31.0005) is gratefully acknowledged.
[1] N. F. Mott, Rev. Mod. Phys. 40, 677 (1968); Metal-Insulator Transitions (Taylor \& Francis, London, 1990); M. Imada, A. Fujimori, and Y. Tokura, Rev. Mod. Phys. 70, 1039 (1998).

[2] G. Kh. Rozenberg, W. Xu, and M. P. Pasternak, Z. Kristallogr. 229, 210 (2014)

[3] V. R. Murthy, Science 253, 303 (1991); I. Jackson, Earth's Mantle: Composition, Structure and Evolution (Cambridge University Press, Cambridge, UK, 1998).
[4] A. F. Goncharov and V. V. Struzhkin, and S. D. Jacobsen, Science 312, 1205 (2006); J.-F. Lin, G. Vankó, S. D. Jacobsen, V. Iota, V. V. Struzhkin, V. B. Prakapenka, A. Kuznetsov, and C.-S. Yoo, ibid. 317, 1740 (2007); H. Ozawa, F. Takahashi, K. Hirose, Y. Ohishi, and N. Hirao, ibid. 334, 792 (2011).

[5] D. G. Isaak, R. E. Cohen, M. J. Mehl, and D. J. Singh, Phys. Rev. B 47, 7720 (1993); R. E. Cohen, I. I. Mazin, and D. G. Isaak, Science 275, 654 (1997). 
[6] M. P. Pasternak, R. D. Taylor, R. Jeanloz, X. Li, J. H. Nguyen, and C. A. McCammon, Phys. Rev. Lett. 79, 5046 (1997); T. Atou, M. Kawasaki, and S. Nakajima, Jpn. J. Appl. Phys. 43, L1281 (2004); A. P. Kantor, S. D. Jacobsen, I. Yu. Kantor, L. S. Dubrovinsky, C. A. McCammon, H. J. Reichmann, and I. N. Goncharenko, Phys. Rev. Lett. 93, 215502 (2004); J.-P. Rueff, A. Mattila, J. Badro, G. Vankó, and A. Shukla, J. Phys.: Condens. Matter 17, S717 (2005); C. S. Yoo, B. Maddox, J.-H. P. Klepeis, V. Iota, W. Evans, A. McMahan, M. Y. Hu, P. Chow, M. Somayazulu, D. Häusermann, R. T. Scalettar, and W. E. Pickett, Phys. Rev. Lett. 94, 115502 (2005); H. Ozawa, K. Hirose, K. Ohta, H. Ishii, N. Hiraoka, Y. Ohishi, and Y. Seto, Phys. Rev. B 84, 134417 (2011); R. A. Fischer, A. J. Campbell, O. T. Lord, G. A. Shofner, P. Dera, and V. B. Prakapenka, Geophys. Res. Lett. 38, L24301 (2011); A. G. Gavriliuk, I. A. Trojan, and V. V. Struzhkin, Phys. Rev. Lett. 109, 086402 (2012).

[7] K. Terakura, T. Oguchi, A. R. Williams, and J. Kübler, Phys. Rev. B 30, 4734 (1984); I. I. Mazin and V. I. Anisimov, ibid. 55, 12822 (1997).

[8] V. I. Anisimov, J. Zaanen, and O. K. Andersen, Phys. Rev. B 44, 943 (1991); V. I. Anisimov, I. V. Solovyev, M. A. Korotin, M. T. Czyżyk, and G. A. Sawatzky, ibid. 48, 16929 (1993).

[9] W. Metzner and D. Vollhardt, Phys. Rev. Lett. 62, 324 (1989); A. Georges, G. Kotliar, W. Krauth, and M. J. Rozenberg, Rev. Mod. Phys. 68, 13 (1996); G. Kotliar and D. Vollhardt, Phys. Today 57(3), 53 (2004).

[10] V. I. Anisimov, A. I. Poteryaev, M. A. Korotin, A. O. Anokhin, and G. Kotliar, J. Phys.: Condens. Matter 9, 7359 (1997);

A. I. Lichtenstein and M. I. Katsnelson, Phys. Rev. B 57, 6884 (1998); G. Kotliar, S. Y. Savrasov, K. Haule, V. S. Oudovenko, O. Parcollet, and C. A. Marianetti, Rev. Mod. Phys. 78, 865 (2006).

[11] S. Y. Savrasov, G. Kotliar, and E. Abrahams, Nature (London) 410, 793 (2001); X. Dai, S. Y. Savrasov, G. Kotliar, A. Migliori, H. Ledbetter, and E. Abrahams, Science 300, 953 (2003).

[12] A. K. McMahan, K. Held, and R. T. Scalettar, Phys. Rev. B 67, 075108 (2003); B. Amadon, S. Biermann, A. Georges, and F. Aryasetiawan, Phys. Rev. Lett. 96, 066402 (2006); N. Lanatá, Y.-X. Yao, C.-Z. Wang, K.-M. Ho, J. Schmalian, K. Haule, and G. Kotliar, ibid. 111, 196801 (2013); J. Bieder and B. Amadon, Phys. Rev. B 89, 195132 (2014); B. Chakrabarti, M. E. Pezzoli, G. Sordi, K. Haule, and G. Kotliar, ibid. 89, 125113 (2014); K. Haule and T. Birol, Phys. Rev. Lett. 115, 256402 (2015); B. Amadon and A. Gerossier, Phys. Rev. B 91, 161103(R) (2015).

[13] G. Keller, K. Held, V. Eyert, D. Vollhardt, and V. I. Anisimov, Phys. Rev. B 70, 205116 (2004); D. Grieger, C. Piefke, O. E. Peil, and F. Lechermann, ibid. 86, 155121 (2012); D. Grieger and F. Lechermann, ibid. 90, 115115 (2014); I. Leonov, V. I. Anisimov, and D. Vollhardt, ibid. 91, 195115 (2015).

[14] J. Kunes, Dm. M. Korotin, M. A. Korotin, V. I. Anisimov, and P. Werner, Phys. Rev. Lett. 102, 146402 (2009).

[15] I. Leonov, N. Binggeli, D. Korotin, V. I. Anisimov, N. Stojić, and D. Vollhardt, Phys. Rev. Lett. 101, 096405 (2008); I. Leonov, Dm. Korotin, N. Binggeli, V. I. Anisimov, and D. Vollhardt, Phys. Rev. B 81, 075109 (2010); J. Kunes, I. Leonov, M. Kollar, K. Byczuk, V. I. Anisimov, and D. Vollhardt, Eur. Phys. J. Spec. Top. 180, 5 (2010).

[16] I. Leonov, A. I. Poteryaev, V. I. Anisimov, and D. Vollhardt, Phys. Rev. Lett. 106, 106405 (2011); Phys. Rev. B 85, 020401(R) (2012); I. Leonov, A. I. Poteryaev, Yu. N. Gornostyrev, A. I.
Lichtenstein, M. I. Katsnelson, V. I. Anisimov, and D. Vollhardt, Sci. Rep. 4, 5585 (2014); V. I. Anisimov, A. S. Belozerov, A. I. Poteryaev, and I. Leonov, Phys. Rev. B 86, 035152 (2012); A. S. Belozerov, I. Leonov, and V. I. Anisimov, ibid. 87, 125138 (2013).

[17] K. Glazyrin, L. V. Pourovskii, L. Dubrovinsky, O. Narygina, C. McCammon, B. Hewener, V. Schünemann, J. Wolny, K. Muffler, A. I. Chumakov, W. Crichton, M. Hanfland, V. B. Prakapenka, F. Tasnadi, M. Ekholm, M. Aichhorn, V. Vildosola, A. V. Ruban, M. I. Katsnelson, and I. A. Abrikosov, Phys. Rev. Lett. 110, 117206 (2013); L. V. Pourovskii, J. Mravlje, M. Ferrero, O. Parcollet, and I. A. Abrikosov, Phys. Rev. B 90, 155120 (2014).

[18] S. Y. Savrasov, K. Haule, and G. Kotliar, Phys. Rev. Lett. 96, 036404 (2006).

[19] M. Aichhorn, L. Pourovskii, and A. Georges, Phys. Rev. B 84, 054529 (2011).

[20] H. Park, A. J. Millis, and C. A. Marianetti, Phys. Rev. B 89, 245133 (2014).

[21] I. Leonov, V. I. Anisimov, and D. Vollhardt, Phys. Rev. Lett. 112, 146401 (2014).

[22] J. Kunes, A. V. Lukoyanov, V. I. Anisimov, R. T. Scalettar, and W. E. Pickett, Nat. Mater. 7, 198 (2008); J. Kunes and V. I. Anisimov, Ann. Phys. (Berlin) 523, 682 (2011).

[23] A. O. Shorikov, Z. V. Pchelkina, V. I. Anisimov, S. L. Skornyakov, and M. A. Korotin, Phys. Rev. B 82, 195101 (2010).

[24] K. Ohta, R. E. Cohen, K. Hirose, K. Haule, K. Shimizu, and Y. Ohishi, Phys. Rev. Lett. 108, 026403 (2012).

[25] I. Leonov, Phys. Rev. B 92, 085142 (2015).

[26] L. Huang, Y. Wang, and X. Dai, Phys. Rev. B 85, 245110 (2012); A. A. Dyachenko, A. O. Shorikov, A. V. Lukoyanov, and V. I. Anisimov, JETP Lett. 96, 56 (2012).

[27] X. Ren, I. Leonov, G. Keller, M. Kollar, I. Nekrasov, and D. Vollhardt, Phys. Rev. B 74, 195114 (2006); J. Kunes, V. I. Anisimov, A. V. Lukoyanov, and D. Vollhardt, ibid. 75, 165115 (2007); J. Kunes, V. I. Anisimov, S. L. Skornyakov, A. V. Lukoyanov, and D. Vollhardt, Phys. Rev. Lett. 99, 156404 (2007).

[28] K. Byczuk, J. Kunes, W. Hofstetter, and D. Vollhardt, Phys. Rev. Lett. 108, 087004 (2012); P. Thunström, I. Di Marco, and O. Eriksson, ibid. 109, 186401 (2012).

[29] I. A. Nekrasov, N. S. Pavlov, and M. V. Sadovskii, JETP Lett. 95, 581 (2012); J. Exp. Theor. Phys. 116, 620 (2013).

[30] For a review, see, e.g., L. V. Pourovskii, B. Amadon, S. Biermann, and A. Georges, Phys. Rev. B 76, 235101 (2007); K. Haule, ibid. 75, 155113 (2007); B. Amadon, F. Lechermann, A. Georges, F. Jollet, T. O. Wehling, and A. I. Lichtenstein, ibid. 77, 205112 (2008); M. Aichhorn, L. Pourovskii, V. Vildosola, M. Ferrero, O. Parcollet, T. Miyake, A. Georges, and S. Biermann, ibid. 80, 085101 (2009); B. Amadon, J. Phys.: Condens. Matter 24, 075604 (2012); H. Park, A. J. Millis, and C. A. Marianetti, Phys. Rev. B 90, 235103 (2014).

[31] S. Baroni, S. de Gironcoli, A. Dal Corso, and P. Giannozzi, Rev. Mod. Phys. 73, 515 (2001); P. Giannozzi, S. Baroni, N. Bonini, M. Calandra, R. Car et al., J. Phys.: Condens. Matter 21, 395502 (2009).

[32] N. Marzari and D. Vanderbilt, Phys. Rev. B 56, 12847 (1997); N. Marzari, A. A. Mostofi, J. R. Yates, I. Souza, and D. Vanderbilt, Rev. Mod. Phys. 84, 1419 (2012). 
[33] V. I. Anisimov, D. E. Kondakov, A. V. Kozhevnikov, I. A. Nekrasov, Z. V. Pchelkina, J. W. Allen, S.-K. Mo, H.-D. Kim, P. Metcalf, S. Suga, A. Sekiyama, G. Keller, I. Leonov, X. Ren, and D. Vollhardt, Phys. Rev. B 71, 125119 (2005); G. Trimarchi, I. Leonov, N. Binggeli, Dm. Korotin, and V. I. Anisimov, J. Phys.: Condens. Matter 20, 135227 (2008); Dm. Korotin, A. V. Kozhevnikov, S. L. Skornyakov, I. Leonov, N. Binggeli, V. I. Anisimov, and G. Trimarchi, Eur. Phys. J. B 65, 91 (2008).

[34] P. Werner, A. Comanac, L. de'Medici, M. Troyer, and A. J. Millis, Phys. Rev. Lett. 97, 076405 (2006); E. Gull, A. J. Millis, A. I. Lichtenstein, A. N. Rubtsov, M. Troyer, and P. Werner, Rev. Mod. Phys. 83, 349 (2011).
[35] J. M. Tomczak, T. Miyake, and F. Aryasetiawan, Phys. Rev. B 81, 115116 (2010); K. Karlsson, F. Aryasetiawan, and O. Jepsen, ibid. 81, 245113 (2010); R. Sakuma and F. Aryasetiawan, ibid. 87, 165118 (2013).

[36] We note, however, that the calculated transition pressure $P_{c}$ depends very sensitively on the choice of the interaction parameters $U$ and $J$. In particular, for $\mathrm{MnO}, P_{c} \sim 132.9 \mathrm{GPa}$ for $U=7 \mathrm{eV}$ and $J=0.86 \mathrm{eV}$ and is about $108.8 \mathrm{GPa}$ for $U=8$ $\mathrm{eV}$ and $J=0.75 \mathrm{eV}$, respectively. For $\mathrm{FeO}$, it is about $55 \mathrm{GPa}$ for $U=5 \mathrm{eV}$ and $J=0.89 \mathrm{eV}$, and $\sim 80 \mathrm{GPa}$ for $U=8 \mathrm{eV}$ and $J=0.89 \mathrm{eV}$; for NiO, $\sim 248 \mathrm{GPa}$ for $U=8 \mathrm{eV}$ and $J=1 \mathrm{eV}$.

[37] W. F. Brinkman and T. M. Rice, Phys. Rev. B 2, 4302 (1970). 